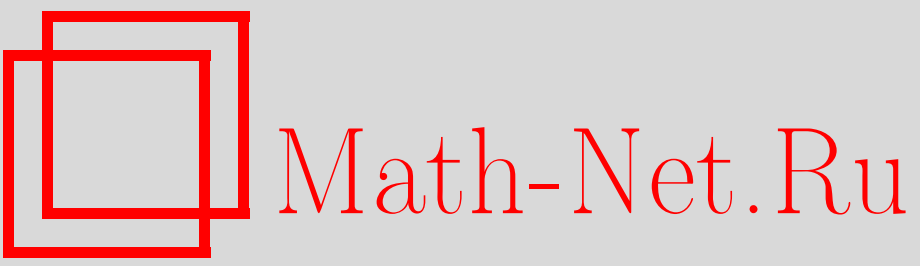

Д. И. Казаков, А. И. Онищенко, Численный анализ сходящейся теории возмущений в квантовой теории, ТМФ, 1997, том 110, номер 2, 291-297

DOI: https://doi.org/10.4213/tmf968

Использование Общероссийского математического портала Math-Net.Ru подразумевает, что вы прочитали и согласны с пользовательским соглашением

http://www . mathnet.ru/rus/agreement

Параметры загрузки:

IP: 54.172 .240 .79

26 апреля 2023 г., 16:16:59 
ТЕОРЕТИЧЕСКАЯ

И МАТЕМАТИЧЕСКАЯ

ФИЗИКА

Том 110, № 2

февраль, 1997

Д. И. Казаков* , А.И. Онищенко

\section{ЧИСЛЕННЫЙ АНАЛИЗ СХОДЯЩЕЙСЯ ТЕОРИИ ВОЗМУШЕНИЙ В КВАНТОВОЙ ТЕОРИИ}

Представлены результаты численного анализа сходимости нового ряда теории возмущений, предложенного в [1]. Рассмотрены два примера: ангармонический осциллятор в квантовой механике и ренормгрупповая бета-функция в теории поля. Показано, что в первом случае ряд действительно сходится к точному значению в широком интервале значений параметра разложения. Этот интервал можно расширить с помощью паде́-аппроксимации. В случае теории поля результаты имеют более сильную зависимость от параметра регуляризации. Обсуждается алгоритм выбора этого параметра, позволяющий получать устойчивые результаты.

\section{1. ВВЕДЕНИЕ}

Хорошо известно, что ряды теории возмушений в квантовой механике и квантовой теории поля являются асимптотическими [2]. В недавней работе [1] авторы предложили метод построения новой сходящейся теории возмушений. Они доказали, что новые ряды, будучи модификашией обычной теории возмушений, являются сходяшимися и могут служить основой для приближенного вычисления функциональных интегралов в нелинейных задачах квантовой физики.

Целью настоящей заметки является демонстрация того, как предложенный алгоритм работает при наличии ограниченного числа вычисленных членов ряда. Мы рассмотрели два характерных примера: основной уровень энергии ангармонического осциллятора и $\beta$-функцию в скалярной 4-мерной теории поля. В обоих случаях мы вычислили несколько членов нового разложения и провели численный анализ его сходимости.

\section{2. НОВАЯ ТЕОРИЯ ВОЗМУЩЕНИЙ}

Выпишем необходимые формулы из [1] для случая, когда взаимодействие имеет вид $g \varphi^{4}$.

Для ангармонического осциллятора энергия основного состояния может быть представлена в виде функционального интеграла

$$
E_{0}(g)=-\lim _{T \rightarrow \infty} \frac{1}{T} \log \frac{\int d x \exp \left[-\int_{0}^{T} d t\left(\dot{x}^{2} / 2+x^{2} / 2+g x^{4}\right)\right]}{\int d x \exp \left[-\int_{0}^{T} d t\left(\dot{x}^{2} / 2\right)\right]},
$$

\footnotetext{
* Объединенный институт ядерных исследований

${ }^{\dagger}$ Московский физико-технический институт
} 
в то время как в теории поля $\beta$-функция получается из четырех- и двухточечных функций Грина

$$
G_{n}\left(x_{1}, \ldots, x_{n}\right)=\frac{\int \delta \varphi \exp \left\{-\int S[\varphi]\right\} \varphi\left(x_{1}\right) \cdots \varphi\left(x_{n}\right)}{\int \delta \varphi \exp \left\{-\int S[\varphi]\right\}}
$$

где $S[\varphi]=S_{0}[\varphi]+g \int \varphi^{4} d x$.

Обычная теория возмущений для интересующих нас величин имеет вид асимптотического разложения

$$
F(g)=\sum_{k}^{\infty}(-g)^{k} F_{k}
$$

где коэффициенты $F_{k}$ выражаются через функциональные интегралы

$$
F_{k} \sim \frac{\int \delta \varphi e^{-S_{0}}\left[\int d x\left(\varphi^{4}\right)\right]^{k}}{k ! \int \delta \varphi e^{-S_{0}}}
$$

и ведут себя как

$$
F_{k} \sim k ! k^{b} a^{k} \text { при } k \rightarrow \infty .
$$

Новое разложение [1], наоборот, является сходящимся и выражается в нашем случае в форме двух рядов

$$
F(g)=\sum_{k=0}^{\infty} \frac{k !}{4 k !} g^{k} A_{4 k}(R) F_{k}+\sum_{k=0}^{\infty} \frac{k !}{(4 k+2) !} g^{k+1 / 2} A_{4 k+2}(R) F_{k+1 / 2},
$$

где коэффициенты $A_{n}$ имеют вид

$$
A_{n}(R)=\frac{1}{\pi} \int_{-\infty}^{\infty} \frac{d^{n}}{d r^{n}} e^{-r^{4}} \frac{\sin R r}{r} d r
$$

и зависят от параметра регуляризации $R$, а функциональные интегралы $F_{n}$ даются формулой (4).

Здесь необходимо сделать два замечания:

Во-первых, как показано в [1], выбирая параметр $R$ достаточно большим, можно получить сходяшийся ряд и аппроксимировать правильный результат с желаемой точностью при условии, что число членов ряда достаточно велико. С другой стороны, если $R \rightarrow \infty$, то мы возврашаемся к исходному ряду обычной теории возмушений.

Во-вторых, функциональный интеграл $F_{k+1 / 2}$ несколько необычен, т.к. содержит квадратный корень из взаимодействия. Хотя авторы и предложили некоторую процедуру для преодоления этой трудности, они не построили явный алгоритм для вычислений. Для решения этой технической проблемы мы используем неравенства Соболева, справедливые для функциональных интегралов в перенормированной теории [3] (мы предполагаем, что все необходимые формулы соответствуюшим образом ренормированы). Неравенство Соболева для взаимодействия $\varphi^{4}$ имеет вид

$$
\sqrt{\int d x \varphi^{4}} \leq C_{S} \int d x \frac{1}{2}\left[(\partial \varphi)^{2}+m^{2} \varphi^{2}\right]
$$


где $C_{S}$ - константа Соболева, равная $\sqrt{3} / 2$ для осциллятора $(D=1)$ и $\frac{1}{2 \pi} \sqrt{\frac{3}{2}}$ для теории $\varphi^{4}$ при $D=4$.

Используя это неравенство, можно избавиться от квадратного корня в (5) и заменить его кинетическим членом, получая таким образом оценку сверху. Это позволяет выразить новые члены ряда через уже известные и построить новое разложение, не производя реальных вычислений.

\section{3. ВЫБОР ПАРАМЕТРА РЕГУЛЯРИЗАЦИИ $R$}

Коэффициенты $A_{n}(R)$ уравнения (6) имеют сложную зависимость от параметра регуляризации $R$. Мы исследовали их поведение при различных значениях $R$ и $n$ и обнаружили следующую общую черту: при фиксированном номере $n$ коэффициенты $A_{n}(R)$ являются сильно осциллирующими функциями $R$, причем сначала идет область малых осцилляций, затем область осцилляций с большой амплитудой, после чего осцилляции опять затухают и коэффициенты выходят на свои асимптотические значения

$$
A_{4 k}(\infty)=\frac{(-)^{k}}{k !}(4 k) !, \quad A_{4 k+2}(\infty)=0 .
$$

На рис. 1 приведен характерный пример поведения коэффициента $A_{14}$, нормированного на асимптотическое значение 14 ! 3 !. С увеличением номера $n$ область осцилляций сдвигается в сторону бо́льших $R$ и их амплитуда возрастает.

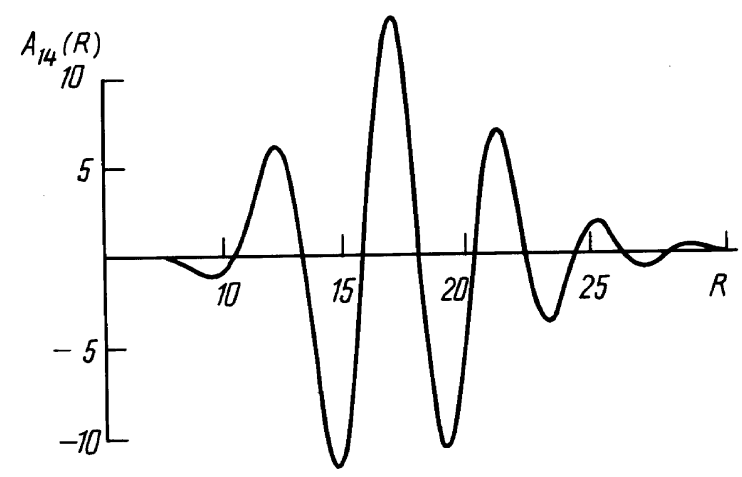

Рис.1. Зависимость коэффициентов $A_{n}(R)$ от параметра $R, n=14$.

С другой стороны, если зафиксировать значение $R$, то зависимость от $n$ также представляет собой сильно осциллируюшую кривую примерно такого же вида, показанную на рис. 2 . При увеличении $R$ область максимума также сдвигается вправо.

Для получения быстросходящегося ряда желательно иметь убываюшие коэффициенты. Поэтому для данного значения $R$ необходимо иметь достаточно много членов ряда - область I на рис. 2. В этом случае отброшенные члены ряда малы и дают лиш малую поправку к результату. Конкретное же значение параметра $R$ несушественно при условии, что оно достаточно велико, чтобы ряд сходился к правильному значению и необходимое число членов ряда имелось в наличии. 


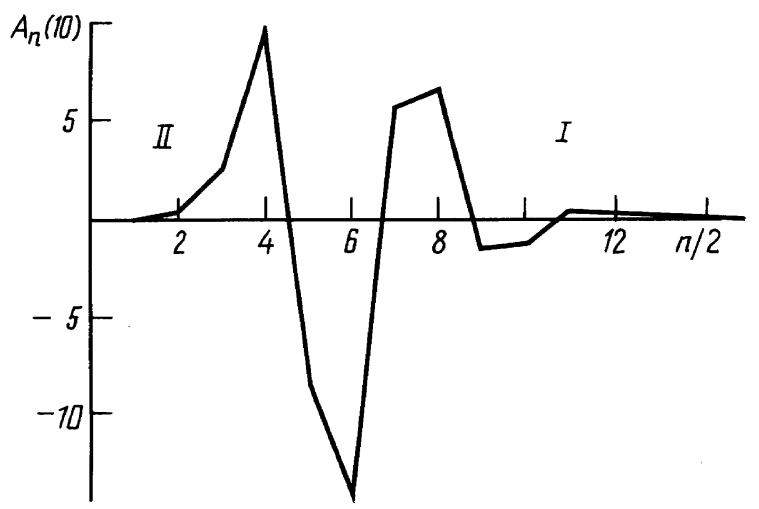

Рис.2. Зависимость коэффициентов $A_{n}(R)$ от параметра $n, R=10$.

Практическая реализация этой процедуры, однако, имеет тот недостаток, что для достижения приемлемой точности $R$ должно быть достаточно большим и, следовательно, число членов ряда достаточно велико.

Альтернативным вариантом является выбор параметров $R$ и $n$, соответствующих области до максимума - область II на рис. 2. В этом случае число членов ряда достаточно мало, однако зависимость результата от выбора параметра $R$ становится существенной, т.к. с ростом $n$ мы попадаем в область осцилляций коэффициентов $A_{n}(R)$. Поэтому при фиксированном числе членов ряда необходима тонкая подстройка параметра $R$.

В дальнейшем мы определяли параметр $R$ из условия минимизации относительного вклада последнего учтенного члена ряда. В случае взаимодействия вида $\phi^{4}$ это означает минимизацию следуюшего выражения:

$$
\min _{R} \Delta_{N}=\min _{R} \frac{A_{N}(R) F_{N g^{N}} N ! /(4 N) !+A_{N+2}(R) F_{(N+1 / 2) g^{N+1 / 2}} N ! /(4 N+2) !}{\Sigma_{N-1}},
$$

где параметр разложения $g$ может быть достаточно произвольным и определять область применимости полученного разложения. Такой критерий выбора параметра $R$ соответствует минимизации относительной погрешности при отбрасывании последующих членов ряда.

Ниже мы продемонстрируем сходимость получающегося разложения на конкретных примерах из квантовой теории: ангармонического осциллятора в квантовой механике и скалярной теории поля со взаимодействием $\phi^{4}$.

\section{4. ЧИСЛЕННЫЙ АНАЛИЗ ПРИ ОГРАНИЧЕННОМ ЧИСЛЕ ЧЛЕНОВ РЯДА}

4.1. Квантовая механика: ангармонический осциллятор. Ангармонический осциллятор со взаимодействием вида $g x^{4}$ служит эталонным примером для численных экспериментов. С одной стороны, эту модель можно рассматривать как теорию поля в одном измерении, а с другой, для нее известно решение, следующее из численного интегрирования уравнения Шредингера. 


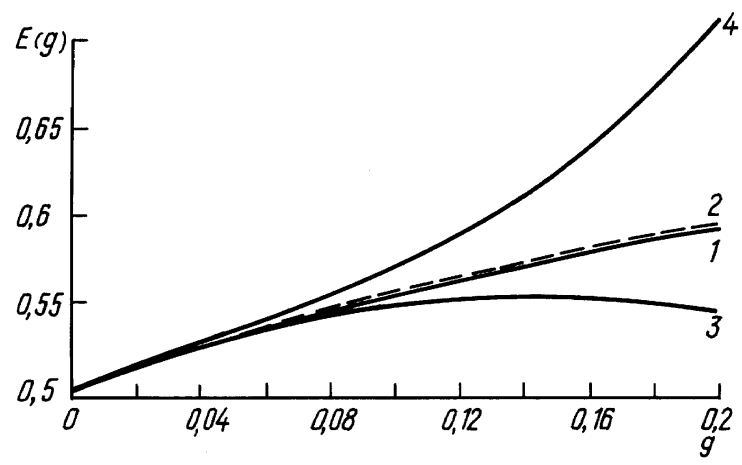

РИс.3. Основной уровень энергии ангармонического осциллятора. Сплошная кривая 1 численное решение уравнения Шредингера, штриховая кривая 2 - новая теория возмущений, кривые 3 и 4 - второй и третий порядок обычной теории возмущений, соответственно.

Рассмотрим в этой модели задачу вычисления основного уровня энергии. Она дается функциональным интегралом вида (1) и разлагается в асимптотический ряд по константе связи типа (3):

$$
E_{0}(g)=\frac{1}{2}-\sum_{k=1}^{\infty}(-g)^{k} E_{k}
$$

причем известно довольно большое число членов ряда [4]:

$$
E_{1}=\frac{3}{4}, \quad E_{2}=\frac{21}{8}, \quad E_{3}=\frac{333}{16}, \quad E_{4}=\frac{30885}{128}, \quad E_{5}=\frac{916731}{256}, \ldots
$$

В дальнейшем мы ограничимся несколькими первыми членами ряда. Тонкая подстройка параметра $R$ согласно сформулированному выше критерию минимизации относительной погрешности дает следуюшее значение $R=20.305$ при $g=1$ и при учете трех членов исходного ряда. Минимизация $\Delta_{N}$ при учете двух или четырех членов ряда несушественно меняет это значение, хотя количество знаков после запятой показывает насколько тонкой является подстройка параметров.

Вычисляя с помощью (6) коэффициенты $A_{n}(R)$ и используя неравенство Соболева (7), построим новое сходящееся разложение (5). Полученная таким образом энергия основного состояния ангармонического осциллятора как функция константы связи представлена на рис. 3. Там же для сравнения показана кривая, соответствуюшая численному решению уравнения Шредингера и первым членам обычной теории возмущений.

Как следует из рис. 3 , мы наблюдаем хорошее согласие нашего решения с точным решением вплоть до значения константы связи $g=0.2$. В то же время обычная теория возмушений начинает расходиться гораздо раньше.

Имея сходяшийся ряд, можно улучшить свойства его сходимости, применив паде́-аппроксимацию. В нашем случае, имея 3 члена исходного ряда и соответственно 6 членов нового ряда, построим паде́-аппроксимант [3,3]. Фитирование параметра $R$ при этом дает $R=20.325$. Результат представлен на рис. 4 . Как видно, использование паде́-аппроксимации позволяет расширить гранищы применимости нового ряда теории возмущений вплоть до значений $g=0.5$. 


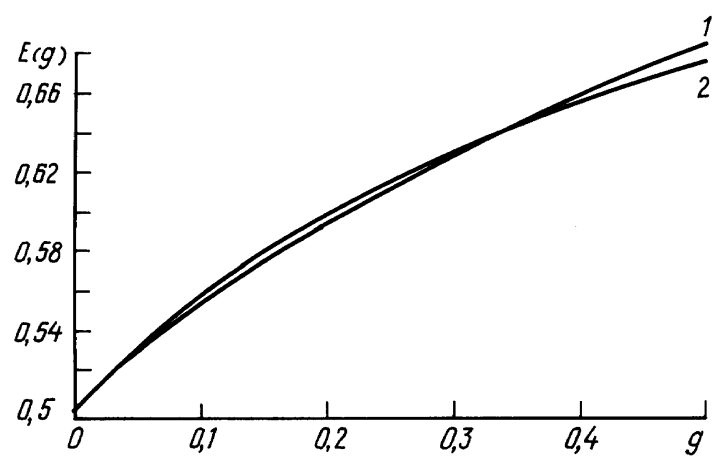

Рис.4. Основной уровень энергии ангармонического осциллятора: кривая 1 - численное решение уравнения Шредингера, кривая 2 - паде́-аппроксимация новой теории возмущений.

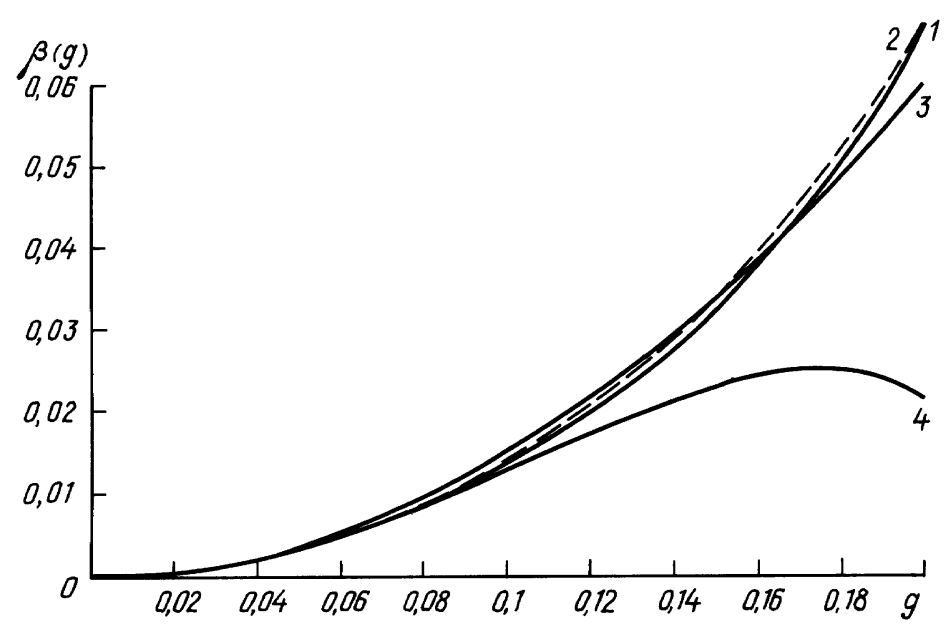

Рис.5. $\beta$-функция в теории $\phi^{4}$. Сплошная кривая 1 - новая теория возмушений (четыре петли), штриховая кривая 2 - новая теория возмущений (три петли), кривые 3 и 4 - обычная теория возмущений в двух и четырех петлях, соответственно.

4.2. Квантовая теория поля: $\beta$-функция в теории $\phi^{4}$. Применим тот же прием для вычисления ренормгрупповой $\beta$-функции в скалярной теории поля со взаимодействием вида $16 \pi^{2} / 4 ! \phi^{4}$. $\beta$-функция получается дифференцированием четырех- и двухточечных функций Грина (2) по точке нормировки. Асимптотический ряд теории возмущений имеет вид

$$
\beta(g)=\sum_{n=2}^{\infty}(-g)^{n} \beta_{n}, \quad \beta_{2}=\frac{3}{2}, \quad \beta_{3}=\frac{17}{6}, \quad \beta_{4} \approx 19.3, \quad \beta_{4} \approx 146, \ldots
$$

Заметим, что в отличие от случая ангармонического осциллятора, в теории поля вычисление коэффициентов $\beta$-функции представляет собой весьма сложную задачу $[2,5]$.

Используя неравенство Соболева $(7)$, получаем коэффициенты $A_{n}(R)$ и находим параметр $R$ из условия минимизации погрешности. Он оказывается равным 37.35. Полученная таким образом зависимость $\beta$-функции от константы связи в случае учета трех 
и четырех членов исходного ряда представлена на рис. 5. Здесь мы не имеем эталонного точного решения, с которым можно было бы проводить сравнения, но видим, что четырехпетлевое приближение практически не отличается от трехпетлевого до значений константы $g \approx 0.2$; это можно трактовать так, что мы имеем хорошее приближение к точному решению в этой области. Этот результат находится также в хорошем согласии с результатами, полученными суммированием асимптотического ряда обычной теории возмушений с использованием асимптотических оценок [2].

\section{5. ЗАКЛЮЧЕНИЕ}

Наша цель состояла в том, чтобы продемонстрировать, как предложенная в [1] новая сходящаяся теория возмушений работает в случае, когда число вычисленных членов ряда невелико. Это соответствует реальной ситуации в квантовой теории поля. Эталонный пример ангармонического осциллятора показывает, что новая теория возмушений действительно дает удовлетворительное описание. В то же время при выборе параметра регуляризации $R$ из области до максимума (область II на рис. 2 ) зависимость от $R$ оказывается сильной и требует тонкой подстройки, внося дополнительный произвол. При этом влияние отброшенных членов ряда теории возмущений может быть велико, что не позволяет рассматривать большие значения параметра разложения при восстановлении функции. Тем не менее последовательное проведение предложенной процедуры позволяет работать со сходящимся рядом теории возмушений и получать достоверные результаты.

Авторы признательны В.В.Белокурову и И. Л. Соловцову за полезные обсуждения.

\section{Список литературы}

[1] В. В. Белокуров, Ю. П. Соловьев, Е. Т. Шавгулидзе. ТМФ. 1996. Т. 109. № 1. С. 51-69; Препринт НИИЯФ МГУ 95-31/395, 95-32/396.

[2] D. I. Kazakov, D. V. Shirkov. Fortschr. Phys. 1980. B. 28. S. 465.

[3] J. Zinn-Justin. Quantum Field Theory and Critical Phenomena. Oxford: Clarendon Press, 1989. P. 814.

[4] C. M. Bender, T. T. Wu. Phys. Rev. D. 1972. V. 7. P. 1620.

[5] K. G. Chetyrkin, S. G. Gorishny, S. A. Larin, F. V. Tkachov. Phys. Lett. B. 1983. V. 132. P. 351; D. I. Kazakov. Phys. Lett. B. 1983. V. 133. P. 406.

Поступила в редакцию 5.VIII.1996 г.

\section{I. Kazakov, A.I. Onitchenko NUMERICAL ANALYSIS OF CONVERGENT
PERTURBATION THEORY IN QUANTUM FIELD THEORY}

Results of numerical analysis of convergency for a new series of perturbation theory are presented. Two examples are considered: anharmonic oscillator in quantum mechanics and the renormalization group $\beta$-function in field theory. It is shown that in the former case the series converges to an exact value in the wide region of the expansion parameter. This region can be enlarged by using the Padé approximation. In the field theory case the results have the stronger dependence on the expansion parameter. An algorithm of choosing this parameter in such a way as to obtain stable results is discussed. 